\title{
ESTRUCTURA SEMÁNTICA Y ANÁLISIS IDEOLÓGICO
}

\author{
José Miguel Rodríguez-Zamora
}

\begin{abstract}
RESUMEN
En este artículo se hace una revisión de las principales categorías de la semántica estructural. Particular interés tiene el estudio del concepto de campo semántico como base para un modelo de la semántica estructural con aplicación para el análisis ideológico.

Palabras clave: Semántica, semántica estructural, campo semántico, análisis ideológico.
\end{abstract}

\begin{abstract}
In this article a revision of the main categories of the structural semantics is made. Particular interest has the study of the concept of semantic field like base for a model of the structural semantics with application for the ideological analysis.

Key words: Semantics, Structural Semantics, Semantic Field, Ideological Analysis.
\end{abstract}

\section{Introducción}

La semántica lingüística ha contribuido en el análisis en toda investigación en la que interviene el lenguaje y la comunicación humana; es decir, en la que la racionalidad comunicativa forma parte de la interacción social. Derivado de ello surge el llamado análisis ideológico, el cual es un instrumento imprescindible en el estudio de los procesos psicosociales, en la construcción de representaciones del mundo y las formas de la institucionalidad, entre otros ${ }^{1}$. En general, se puede suscribir la siguiente observación epistemológico-metodológica de Habermas:

Quien se sirve de un lenguaje natural para entenderse con un destinatario acerca de algo en el mundo, se ve obligado a adoptar una actitud realizativa y a comprometerse con determinadas suposiciones. Entre otras cosas, tiene que partir de que los participantes persiguen sin reservas sus fines ilocucionarios, ligan

Dr. José Miguel Rodríguez Zamora. Profesor de la Escuela de Ciencias Políticas, Universidad de Costa Rica. San Pedro de Montes de Oca, San José, Costa Rica.

Recepción: 21-6-04

Aceptación: 24-6-04 
su acuerdo al reconocimiento de pretensiones de validez susceptibles de críticas y se muestran dispuestos a asumir las obligaciones relevantes para la secuencia de interacción que siguen de un consenso. La racionalidad comunicativa se manifiesta en una trama descentrada de condiciones trascendentalmente posibilitantes, formadoras de estructuras, y que impregnan la interacción, pero no es una facultad subjetiva que dicta a los actores qué es lo que deben hacer" (Habermas 1998: 66).

Por ello, conviene definir el análisis ideológico. Este puede ser entendido como un método de estudio de las estructuras mentales y de las representaciones que realizan los actores socio-políticos. La investigación contemporánea privilegia un enfoque interdisciplinario, no restrictivo y más comprensivo. Sin embargo, esto no excluye la aproximación propiamente desde la semántica lingüística stricto senso. Todo lo contrario, la metodología comprensiva supone desde su base analítica una semántica del discurso, que a su vez, encuentra su origen en la semántica estructural ${ }^{2}$. De aquí surgen varias preguntas: ¿Qué papel desempeña la semántica estructural en este proceso? ¿Qué importancia tiene para la aplicación del análisis ideológico? Estas son las cuestiones que se responderán en este artículo.

\section{El significado y la estructura}

Para aclarar la relación que existe entre el análisis ideológico y la semántica (teoría del significado), conviene iniciar este estudio con una determinación conceptual. El análisis ideológico trata de establecer un significado al texto, al discurso o a un hecho socio-político. Es decir, que el texto adquiere su significado (semántica) dentro de la estructura linguiística, como se verá más adelante, porque forma parte de un conjunto interrelacionado de representaciones, un imaginario social o colectivo ${ }^{3}$. Este imaginario le otorga el significado lingüístico dentro del conjunto social, es decir, dentro de una estructura o sistema. ¿Cómo se establece este significado? ¿Qué papel desempeña la estructura?

El análisis ideológico nace de la consideración usual tripartita de pre-texto, texto y con-texto. Por pre-texto se entienden las condiciones previas a la elaboración del texto o discurso, es decir, su motivación inmediata y sus condiciones de posibilidad; el texto es el elemento concreto sobre el cual se ejerce el análisis; y el contexto es el conjunto de circunstancias socio-políticas que condicionan -o determinan - las categorías significativas del texto. Dicho análisis estudia, en primer lugar, el contexto, y dentro de este, inserta la investigación inmanente del texto como unidad de análisis, de tal manera que el texto siempre es contextual, ya que necesariamente es parte de una estructura socio-política, o si se quiere, de una cosmovisión histórica. Por ello es importante tener claro qué se entiende por estructura.

En primer término, se puede partir de la clásica metáfora de la red. Según esta analogía, los conceptos y los signos lingüísticos están unidos entre sí de forma integrada semejante a los elementos que componen una red. Afinando más este concepto, se puede suscribir la siguiente definición de estructura:

\footnotetext{
Una entidad autónoma de dependencias internas; o dicho con otras palabras, una totalidad constituida por elementos, hechos o fenómenos relacionados entre sí, cuyo valor o entidad depende de la relación que mantiene cada uno de ellos con los demás. Lo importante de una estructura no son, por tanto, los elementos sino el conjunto de relaciones coherentes que estos mantienen entre sí, ya que en algunas escuelas del estructuralismo la existencia de los elementos no tiene otra finalidad que la de ser los términos de las relaciones (Alcaráz Varó, M. Martínez Linares 1997: 28).
} 
Como es sabido, el término estructura y estructuralismo son tanto polisémicos como polivalentes ${ }^{4}$. Son polisémicos en tanto designan (denotan) diversos conceptos; y polivalentes, en cuanto se refieren a diversos niveles conceptuales. Al respecto, David Lyons (1989) ha hecho notar que el término estructuralismo ha adquirido diferentes significados dependiendo de la orientación lingüística y de la tradición específica. En particular, hay que destacar la idea de que la semántica estructural se compone de una estructura compleja que constituye operacionalmente los elementos constitutivos, las relaciones integradoras y las propiedades emergentes.

Esta definición establece una categorización de elementos que componen la estructura; sin embargo, deja por fuera un concepto fundamental. En efecto, para el estructuralismo es esencial que cada uno de los elementos que forman la estructura guarde relaciones, no sólo de diferenciación, sino también de oposición con otro u otros miembros de la estructura. Oposición es, entonces, una determinación básica de la categoría de estructura; la noción de estructura supone percibir diferencias y relaciones entre términos; es decir, elementos o términos en relaciones. En breve, cuando se habla de estructura se está mencionando: a) un conjunto específico de elementos; b) un sistema de relaciones; c) una totalidad emergente de los anteriores. El siguiente esquema lo ilustra gráficamente:

$$
\begin{aligned}
& {[+]======[-]====[x]} \\
& {[+] \# \# \# \# \# \#[-] \# \# \# \# \#[y]}
\end{aligned}
$$

Donde [+], [-], [x], [y] son los elementos constituyentes de (E); ===, \#\#\# son los tipos de relaciones $(\mathrm{R})$; y $\{$ \} conforma la totalidad de la estructura $(\mathrm{T})$. La estructura, puede decirse grosso modo, es igual a: $(\mathrm{E})+(\mathrm{R})+(\mathrm{T})^{5}$. Por otra parte, se puede admitir que el análisis ideológico construye un modelo de interpretación semántica; de tal modo que en un nivel muy abstracto pueda hablarse de un único modelo estructural con rasgos determinados ${ }^{6}$.

El modelo es una representación teórica de una parte de la realidad lingüístico-ideológica con un objetivo o finalidad determinada. El estructuralismo es, en sí mismo, un modelo general y paradigmático de la investigación linguiística; pero, además, permite la construcción de micromodelos sobre una parte de la realidad ${ }^{7}$. La construcción del modelo se inicia con la propia noción de estructura, ya que el concepto de semántica remite de forma directa al concepto-categoría de estructura. ¿Cuál es este significado y cómo se relaciona con el análisis ideológico?

\section{Desarrollo y características del análisis estructural}

El desarrollo de una teoría coherente del significado se origina en la lingüística estructural. En este caso, como en muchos, la historia se remonta a los trabajos de Ferdinand de Saussure $^{8}$. Es sabido que en su obra fundamental, Curso de lingüística general (1916) ${ }^{9}$, aunque habla de semántica como la ciencia del significado de las palabras, no desarrolla una teoría semántica propiamente dicha. Pero hay en su obra importantes y sugerentes afirmaciones (de Saussure 1983: 146, 148). Este punto daría origen a las llamadas dicotomías o polaridades en los elementos lingüísticos. 
Asimismo, cabe considerar un principio básico de de Saussure. En efecto, para él los términos son menos importantes que las relaciones -la forma de las relaciones- que unen a los elementos. El sistema puede ser considerado como una totalidad compuesta de elementos interrelacionados; el movimiento o desplazamiento de uno de ellos supone el desplazamiento de otro dando así una nueva configuración del sistema. De esta manera, se entiende por estructura la forma en que los diversos elementos se relacionan entre sí en un todo determinado. El propio Saussure pone como ejemplo el caso de juego de ajedrez, en el cual cada una de las movidas establece una nueva configuración del todo (de Saussure 1983: 89). En el lenguaje, las diversas estructuraciones sucesivas son producto del propio lenguaje; por eso actúan plenamente en el eje diacrónico; pero también en el sincrónico como conjunto integrado de elementos móviles ${ }^{10}$.

Con los datos anteriores, se elabora el concepto de valor, elemento constitutivo de la lingüística saussureana y que se distingue del significado (de Saussure 1983: 188). El valor, determinado sincrónica y diacrónicamente, está estipulado por la relación que guarda el signo con otro signo. Es una determinación según la oposición dentro del sistema, el cual depende del estado momentáneo del conjunto de sus elementos. El contenido de una palabra sólo puede ser establecida por su relación con otra u otras palabras. Como se ha dicho, su valor surge de la posición que tenga dentro del sistema. Entonces, el valor de un signo está determinado por las oposiciones en que se encuentra; son constituyentes del concepto. Así, significante y significado son los dos componentes del signo lingüístico y, a la vez, los componentes de la significación. Como el lector puede comprender, este concepto de valor y su distinción del signo lingüístico es extraordinariamente importante para el análisis ideológico.

Además, es primordial destacar el hecho de que el sentido surge de esta determinada configuración categorial independientemente de la intencionalidad del sujeto, porque la comunicación se establece merced a la existencia de una lengua, es decir, de un sistema abstracto de signos, que se utiliza en la comunicación. De esta manera, la estructura determina la dirección específica del significado dentro del propio conjunto (Hörman 1982: Cap.V). Este supuesto estructural implicará, décadas después, la distinción en dos niveles: la estructura superficial y la estructura profunda en la semántica generativa (Katz-Fodor 1963: 170210). Sin embargo, el analista de la ideología sabe muy bien que la intencionalidad discursiva no depende sólo de la estructura linguiística, sino de factores extratextuales, en ocasiones determinantes.

Es importante señalar que estas ideas -oposición, estructura, formalización, etc.-—fueron desarrolladas en una línea más rigurosa y lógica por Louis Hjelmslev de la Escuela de Copenhague. Hay que detenerse un momento en este modelo porque ha sido muy influyente tanto en el análisis textual como en el análisis ideológico. Para este lingüista, la distinción entre forma y sustancia sigue siendo esencial. Sin embargo, la concibe de manera distinta. Por el significante entiende la expresión; por significado, el contenido. Hay forma del contenido y forma de la expresión. La forma es la relación de oposición de las unidades; la sustancia, lo que no se opone. Por este motivo, la linguística sólo debe estudiar la forma. En cambio, lo sustancial de la expresión es el significante: el ruido. Los ruidos segmentados son los fonemas; pero en el contenido son los morfemas. De acuerdo con este criterio, la sustancia del contenido es el significado. El objeto de la lingüística es el estudio del fonema y del morfema. El ruido es de la acústica; el significado es de la filosofía y de la historia. Su modelo linguístico se basa en el supuesto de que los enunciados se pueden reducir a fórmulas algebraicas; la 
llamó glosemática: estudio de las formas del lenguaje, es decir, las relaciones de oposición ${ }^{11}$. Esto se resume en el esquema siguiente:

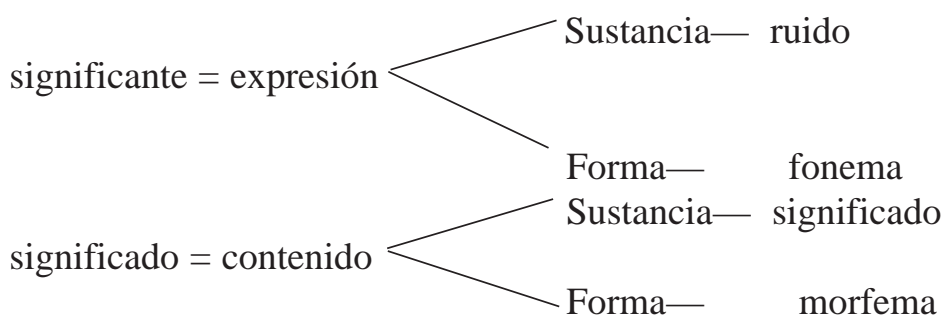

Es interesante comparar este modelo con el presentado por Leonard Bloomfield. En este caso, se explica el significado de acuerdo con los principios conductistas del conocido

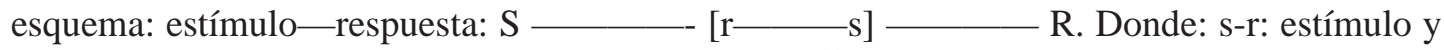
respuesta verbales; S-R: estímulo y respuesta física ${ }^{12}$. De esta forma, el significado de una forma linguiística se establece con base en la situación en que se encuentra el hablante al pronunciarla y la respuesta que suscita en quienes lo escuchan. El concepto o significado de "manzana" corresponde a los estímulos externos que en alguna situación precedente se asocian a la experiencia de lo que es una manzana. Para conocer el significado de un término habría que hacer una lista de todas las situaciones que han motivado ese término. El significado debe entenderse como aquel que es común a todas las formas que pueden ocupar una determinada posición surgida por el mismo estímulo. El significado va a depender irremediablemente del contexto ${ }^{13}$.

En la misma línea de investigación, aparece la famosa hipótesis Wohrf- Sapir, que será la base del relativismo linguiístico y semántico. Edwin Sapir y Benjamín Wohrf, sobre la base de estudios etnolinguísticos sobre el léxico de los colores, el parentesco, animales, plantas, etc., afirmaron que la lengua opera como un filtro entre el hombre y la realidad. En otras palabras, que la lengua sólo permite ver de cierta forma la realidad. De ahí el famoso dictum: "pensamos como nuestra lengua nos permite pensar" (Sapir 1984). En la actualidad, esta hipótesis está muy desacreditada; sin embargo, en su momento sirvió como un factor incitador de la investigación en una línea novedosa para reconocer la existencia de los campos léxicos ${ }^{14}$.

Para el análisis ideológico es importante el concepto de rasgo distintivo. Esto implica que el significado de un término puede descomponerse en rasgos específicos y distintivos (distinctive features, contrastive features) y estos son diferentes según su relación con el significado.

También en la configuración del modelo de la semántica estructural merecen ser mencionados tres modelos del significado. El primero de ellos es de naturaleza referencial y fue propuesto en 1923 por C. K. Ogden y I. A Richards en su obra El significado del significado (1984); el segundo, no referencial, por Stephen Ullmann en Semántica, en el año $1962^{15}$; y el tercero, por Heger en la década del 70. Estos modelos coinciden en proponer una imagen geométrica del significado. Ogden y Richards lo esquematizan de la siguiente forma: 


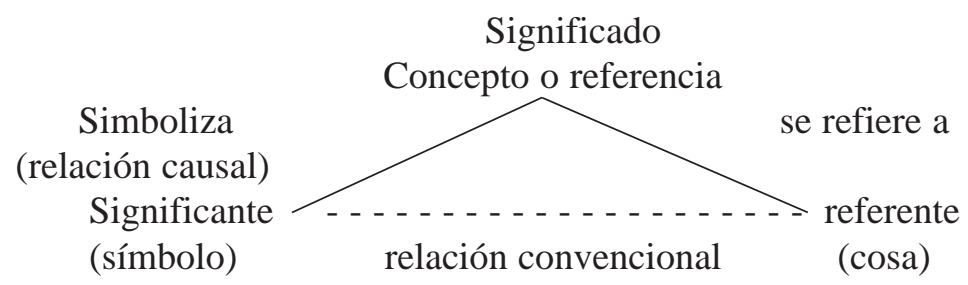

De acuerdo con el gráfico, las relaciones referenciales son aquellas que acuden o se dirigen — señalan — a la realidad externa; las conceptuales no referenciales no acuden a la realidad externa; estas son de naturaleza inmanente a la estructura. Es importante destacar que en este modelo el símbolo (significante) está en relación directa y causal con el pensamiento o referencia; es decir, que el pensamiento está en relación causal de adecuación con el referente considerado como una realidad extralingüística. Posee tres elementos fundamentales: símbolo, concepto y referente.

Más adelante, Ullman establece otro triángulo de relaciones, a saber:

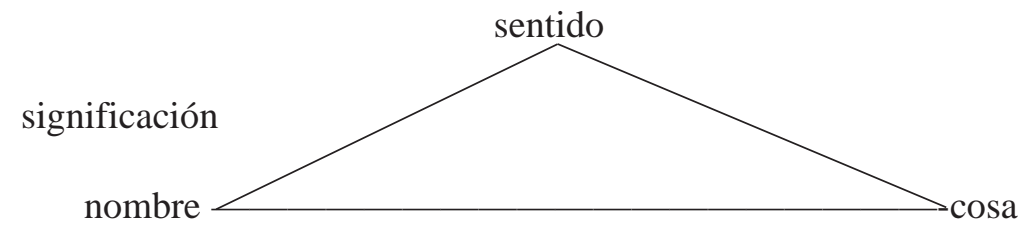

A diferencia del modelo anterior, Ullmann define los términos de la siguiente manera: el nombre es la configuración fonética de la palabra; el sentido es la información que el nombre comunica al oyente; y la cosa es el rasgo, elemento o acontecimiento no lingüístico sobre el que se habla. En consecuencia, la significación es la relación recíproca y reversible entre el nombre y el sentido.

Posteriormente, Heger va a desarrollar mejor el modelo; para ello propone, ya no un triángulo, sino el llamado trapecio semántico (Alcaráz Varó-Martínez Linares 1997: 138). Es un avance frente a los anteriores pues separa de forma nítida lo lingüístico de lo extralingüístico. Dicho trapecio es el siguiente:

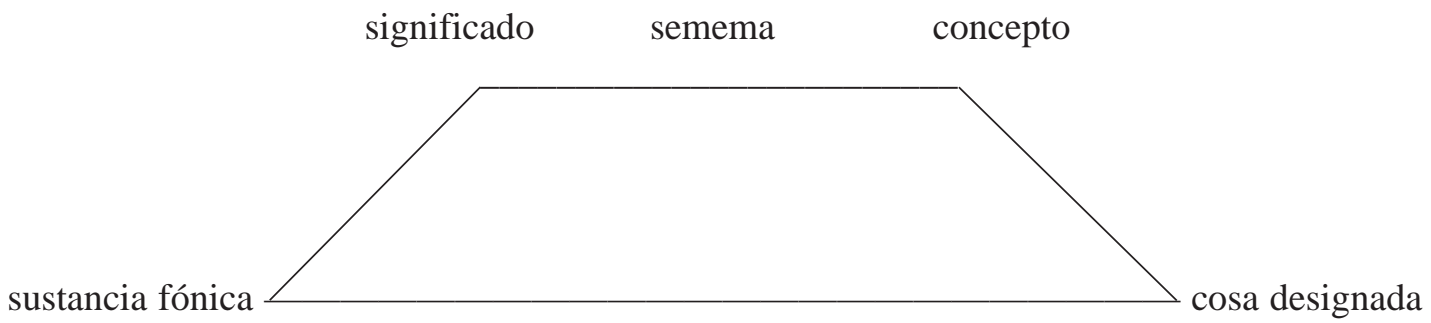

En este caso, el significado es el resultado de una compleja interacción entre el semema (estructura de significación) y el concepto (de naturaleza lingüística); frente a la sustancia fónica y la cosa designada que quedan fuera del ámbito de la lingüística.

Igualmente importante es el modelo estructural desarrollado por Eugenio Coseriu. En efecto, durante la década de los años 60, Coseriu formula una teoría del significado basada en 
los principios estructurales de Saussure, pero ubicándola dentro de un contexto complejo. En este modelo es muy significativa la distinción en cuatro pares de oposiciones: referencialista / no referencialista; conceptualista / no conceptualista; mentalista / no mentalista; contextualista / no contextualista. Esta teoría consiste en la caracterización y el análisis de estructuras lexemáticas; es decir, estructuras en el plano de las unidades de contenido. Se supone que son detectables en el interior de una lengua funcional en particular o en un sector o variedad específica y homogénea de una lengua natural. En consecuencia, es posible aislar sistemas reducidos e inmediatos del léxico, aun formados solamente por dos términos, basados en la oposición. Las oposiciones estructuran el léxico mediante rasgos distintivos ${ }^{16}$.

Siguiendo una línea más formalizada se encuentra el aporte de A. J. Greimas. Este lingüista francés, seguidor de Hjelmslev, es el primero en indicar la posibilidad de la descomposición del contenido de las palabras o morfemas en elementos de contenido ${ }^{17}$. En este caso, los constituyentes semánticos son considerados como unidades de significación combinatoria; los elementos se interrelacionan también de manera formal, pero con un ámbito definido y preciso. Con ello, realiza una contribución esencial para el desarrollo del método y del objeto de la semántica estructural y su aplicación tanto en el análisis de texto como en el análisis ideológico $^{18}$.

Como es sabido, durante la segunda etapa de la gramática generativa en las décadas de los años sesenta y setenta, se despierta un nuevo interés por la semántica debido a que la propuesta de reducir los problemas semánticos a la sintaxis no fue lo fructífero que se esperaba. Particularmente significativo fue el modelo desarrollado por J. Katz en su obra Teoría semántica de 1972 (Katz 1979). En esta obra, Katz intenta construir una semántica basada en los fundamentos propuestos por los nuevos avances de la lingüística transformacional. Para él, una teoría semántica debe dar respuesta a un conjunto específico de preguntas, así como a los problemas fundamentales de la ecuación del significado con la referencia, del significado con el sistema de suposiciones determinantes del uso, del significado de una expresión con la estructura del lenguaje, la definición de las propiedades del modelo semántico y otras cuestiones (Katz 1979: Cap. 2).

En resumen, y como ha señalado el lingüista Berruto, la contribución de los generativistas y postgenerativistas al estructuralismo ha sido valiosa en varios aspectos. En primer término, se trata de explicar la interpretación semántica que damos a las palabras consideradas en su contexto sintáctico como miembros de una clase lingüística (Berruto 1974: Cap. 5). La tarea es la de integrar el tratamiento del significado de las palabras, analizando los rasgos semánticos, dentro de un tratamiento de la organización sintáctica de la frase. Para ello, establecen varios puntos de partida: a) los juicios intuitivos que el hablante está en condiciones de dar respecto de los significados de la frase; b) el análisis de los elementos conceptuales y de las reglas de inserción que constituyen la posibilidad de uso de la palabra de la frase. Pero, además, y sin proponérselo, posibilitó el tránsito del análisis de la frase y del texto, al discurso y al contexto ideológico.

\section{El campo semántico y el método de análisis}

El modelo de Katz, estudiado anteriormente, inspiró el desarrollo de varias líneas de investigación que abrieron la noción del campo. Particular importancia tienen para el análisis 
ideológico las investigaciones de M. Bierwisch. Aunque parte de los mismos principios del modelo semántico de Katz, su trabajo incorpora el uso de elementos de la lógica formal y de la pragmática. Es decir, que con estas investigaciones el estructuralismo semántico se amplía, pues pasa de la semántica componencial de las palabras para convertirse en semántica componencial de las frases, lo que posteriormente dará origen al análisis del texto ${ }^{19}$. Bierwisch amplía la concepción semántica con elementos tomados de la pragmática. Para ello, comienza por elaborar una teoría de los rasgos semánticos y del análisis componencial, es decir, conformado por el conjunto de rasgos semánticos propios de un campo específico ${ }^{20}$.

Es importante recalcar que lo anterior permite el análisis de frases completas y de textos, de tal modo que los "componentes semánticos" representan categorías o principios según los cuales las situaciones y los objetos reales o los ficticios, percibidos o imaginados, son estructurados y clasificados ${ }^{21}$. Los rasgos semánticos no representan propiedades físicas externas, sino condiciones psicológicas según las cuales los seres humanos tratan su ambiente físico y social. Son símbolos de mecanismos interiores mediante los cuales tales fenómenos son percibidos y conceptualizados" (Berruto 1974: 142). De ahí que los componentes semánticos puedan definirse como "entidades teóricas abstractas representantes de complejos mecanismos y estructuras psicológicas. Es plausible que estos formen un inventario universal usado en maneras particulares por cada lengua".

En resumen, ¿qué se entiende por campo semántico? Lyons (1989: 252) ofrece una contribución valiosa. Este autor dice:

\footnotetext{
En lo que cabría considerar la más extrema versión de la teoría del campo, se establece que el vocabulario, $\mathrm{V}$, de una lengua, es un conjunto cerrado de lexemas, $\mathrm{V}=\{\mathrm{L} 1, \mathrm{~L} 2, \mathrm{~L} 3, \ldots, \ln \}$, que pueden seccionarse en un conjunto de campos léxicos $\{\mathrm{C} 1, \mathrm{C} 2, \mathrm{C} 3, \ldots, \mathrm{Cm}\}$, es decir, divididos en subconjuntos, tal que (i) la intersección de los campos distintos cualesquiera es vacía (no hay ningún lexema que sea miembro de más de un campo), y (ii) la unión de todos los campos en v es igual a $\mathrm{V}$ (no hay ningún lexema que no pertenezca a algún campo) (Lyons 1989: 252).
}

Sin embargo, esta versión extrema no parece ajustarse a la realidad lingüística. Lyons propone en su lugar la posibilidad de que los campos sean abiertos e indeterminados; esto sin perjuicio de la formulación esencial básica del conjunto, a saber:

$$
\mathrm{V}=\{\mathrm{L} 1, \mathrm{~L} 2, \mathrm{~L} 3 \ldots\} ; \text { o bien, } \mathrm{V}=\{\mathrm{L} 1, \mathrm{~L} 2,13, \text { etc. }\} \text { y } \mathrm{CL}=\{\mathrm{Li1} 1, \mathrm{Li} 2, \mathrm{Li} 3 \ldots\}, \text { o bien: CL } 0 \text { \{Li1,Li2,Li3, etc. }\}
$$

En otras palabras, que la indeterminación, el traslape o incluso la ausencia de elementos forman parte integral de la noción de campo semántico. De ahí se deduce, además, que no necesariamente toda unidad del vocabulario pertenezca a un campo léxico y sólo a uno, así como puede existir la posibilidad de que no pertenezca a ninguno, y que la semántica puede operar sin recurrir a ninguna de estas posibilidades. Este aspecto es de extraordinaria importancia para el análisis de discurso e ideológico en el cual se suele operar con categorías abiertas. Esto significa que el investigador debe tener presente la compleja interacción de diversos campos, pues varios elementos puede que se presenten en niveles y estructuras paralelas o superpuestas.

Por otra parte, una explicación complementaria ha sido propuesta por Horst Geckeler ${ }^{22}$. De nuevo, aquí se encuentra el principio de la oposición determinante de los rasgos específicos de cada signo. Entonces, es a partir de la diferencia y de su lugar dentro del conjunto, 
como parte de un todo, que se construye el significado. Por eso puede ofrecer la siguiente definición del campo:

\footnotetext{
Campos son las realidades lingüísticas vivas, situadas entre las palabras individuales y el conjunto del vocabulario, que, en cuanto totalidades parciales, tienen como característica común con la palabra el articularse (ergliedern) y, con el vocabulario, el organizarse (ausgliedern). El grado jerárquico es indiferente (Geckeler 1994: 123).
}

En consecuencia, y siguiendo a Weisgerber, el campo es una estructura, una articulación de elementos de acuerdo con un orden lógico, una jerarquía y una direccionalidad (Geckeler 1994: 125; Weisgerber 1979). El campo apunta siempre fuera de sí mismo. Complementa su modelo con la distinción de varios estratos de articulación: campos unidimensionales, pluridimensionaIes. En realidad, resume estos niveles en tres macrocampos (Grossfeld): campos léxicos del dominio de los fenómenos naturales; campos léxicos del dominio de la cultura material; campos léxicos del dominio de lo espiritual. Estos conceptos, aunque algo imprecisos, permiten una articulación estructural de la función ideológica.

Más adelante, G. Kandler ha propuesto varios rasgos esenciales del concepto de campo que permiten ampliar su poder explicativo y su coherencia lógica para el análisis ideológico. En el campo semántico se da un conjunto de relaciones donde cada término obtiene su sentido en el conjunto; pero, a diferencia del sistema fonológico y morfológico, las relaciones no son necesarias. Los principios que propone son: el principio de la totalidad, el principio de la ordenación, el principio de la determinación recíproca, el principio de la integridad, el principio de la diferenciación, el principio de la ausencia de lagunas (Cit. Geckeler 1959: 136).

Kandler formula el principio de totalidad de la siguiente manera: "el valor semántico de una palabra no se hace comprensible partiendo de la palabra individual; desde el punto de vista del contenido, toda palabra depende del conjunto del campo de palabras emparentadas semánticamente". Por ello, se puede decir que la no aceptación de este principio excluiría la posibilidad de que el plano del contenido de la lengua pudiera tener carácter sistemático, como un "sistema de sistemas" (Coseriu) (Cit. Berruto 1974: 141). El principio de ordenación supone que los contenidos léxicos se adapten entre sí en un sistema bien organizado en el que aparezcan realizadas con regularidad varias dimensiones.

Sin embargo, es muy importante tener en cuenta que el significado de un lexema no sólo se constituye con sus relaciones con las palabras vecinas del campo; porque corresponde al campo léxico un significado global, que puede expresarse mediante el archilexema, pero que no necesita estar determinado lexicalmente en todo el campo léxico. En realidad, la definición del contenido por los componentes del campo léxico proporciona el significado propio de cada lexema (Geckeler 1994: 141).

El principio siguiente que estudia Kandler es el de integridad; es decir, que el propio sistema produce una especie de reajuste continuo y llena los vacíos lexicales para mantener la integridad del propio sistema. Según Geckeler, quien suscribe esta opinión, en el sistema se encuentra el campo íntegro, es decir, los significados se han constituido aquí y se limitan recíprocamente. El principio de la diferenciación es definido por Kandler de la siguiente manera: 
El principio de ausencia de lagunas se deriva de los anteriores. No se refiere a las lagunas en la comprensión del hablante-oyente; es decir, de su procesamiento cognitivo del lenguaje. Más bien se refiere a las lagunas en las estructuras lingüísticas. Existen dos clases de lagunas lingüísticas: lagunas determinables interlingüísticamente y lagunas determinables intralingüísticamente. Las lagunas determinadas interlingüísticamente no afectan al punto de vista funcional (análisis lingüístico inmanente). Una lengua no siempre realiza al mismo tiempo un determinado contenido léxico en las cuatro categorías verbales (adjetivo, sustantivo, verbo, adverbio), como, por ejemplo, el esp. blanco, blancura, blanquear, blancamente. El autor aún señala una tercera posibilidad de la aparición de lagunas intralingüísticas que afecta al vocabulario en su estructuración lexemática, es decir, en los campos léxicos.

Esto conduce al último principio, el de las delimitaciones. Este principio se refiere tanto a los límites de cada uno de los campos, como a los límites (denotación y connotación) de los elementos léxicos dentro de cada uno de los campos específicos. En ocasiones, se encuentra una relación recíproca de campos que se define como la existente entre unidad y archiunidad. A este nivel se resuelven las lagunas por el paso de un plano de la estructuración léxica a otro, al ser cubiertos lexicalmente (Geckeler 1994: 167). Sin embargo, puede producirse un traslape (overlap) de significados que se superponen parcialmente, que se delimitan, enriquecen y confunden mutuamente. En algunos casos, este traslape puede ser un caso de designación múltiple, o, incluso, de neutralización de dos o varios campos.

Al mismo tiempo, Berruto ha destacado que el campo semántico es el conjunto formado por los cohipónimos directos de un mismo supraordinado. El conjunto de los cohipónimos directos de pariente forma el campo semántico del parentesco. El conjunto de los cohipónimos de color (es decir, de todos los términos para los que x es un color) forma el campo semántico del color. No siempre los supraordinales, es decir, aquello por lo que vale "x es y pero no y es x", son lexicados por una palabra; con frecuencia son lexicados por un sintagma. Ej. animal doméstico. Esto desplaza el valor de los campos semánticos hacia un plano conceptual donde el supraordinado que determina la existencia de un campo semántico es una "unidad conceptual" representada lexicalmente por una palabra o por un sintagma, "es decir, designada lingüísticamente mediante una unidad de significado".

En otras palabras, el campo semántico se define como el conjunto de los términos que tienen un grupo de rasgos semánticos en común. En este caso, el archilexema o supraordinado es el término cuyos rasgos semánticos coinciden con aquellos que son comunes a los términos del campo semántico.

\section{Observaciones finales}

El lector que ha seguido la exposición anterior podrá comprender la importancia de varios elementos semánticos para el análisis ideológico. En un primer término, se impone la visión de conjunto, es decir, estructural de los procesos correspondientes a las representaciones mentales, sean estas privadas o públicas. Es decir, que estas no están aisladas sino que se presentan en grupos o conjuntos funcionales de instancias significativas.

El campo semántico implica tener siempre en cuenta que la emergencia de los significados no está dada por los elementos, ni por las simples relaciones, sino que se deriva de un 
proceso continuo y dinámico dentro de un conjunto (contextual). Se puede trabajar con una definición amplia, según la cual el campo semántico es el conjunto de términos cuyo significado se refiere a un concepto común; o con una concepción rigurosa, que define el campo semántico como el conjunto de los co-hipónimos directos de un mismo supraordinado. Sin embargo, el propósito de este artículo, como se señaló al principio, es el de retomar los elementos esenciales de la teoría del campo con el objetivo explícito de obtener instrumentos que permitan la elaboración de una explicación significativa dentro de la semántica estructural.

Por eso lleva razón Habermas al insistir en que todo uso del lenguaje natural implica una actitud realizativa, pues supone una determinada ordenación del lenguaje y de su concepción del mundo. En conclusión, es correcta su afirmación de que: "La racionalidad comunicativa se manifiesta en una trama descentrada de condiciones trascendentalmente posibilitantes, formadoras de estructuras, y que impregnan la interacción, pero no es una facultad subjetiva que dicta a los actores qué es lo que deben hacer" (Habermas 1998: 66), actitud que implica la construcción de un modelo estructurado de significados.

En segundo término, el método estructural permite elevarse hacia la construcción de hipótesis y teorías de gran alcance explicativo sobre los procesos de construcción ideológica del significado. Los diversos constituyentes que se han revisado con cierto detenimiento son elementos de una metodología rigurosa que permite establecer los criterios, niveles, las variables y los parámetros del análisis ideológico. Para la semántica estructural, los rasgos distintivos operan como determinantes del significado en un doble movimiento de oposición y de semejanza. La configuración de una matriz de rasgos implica la existencia de un campo que permite establecer unidades de oposición y de distinción. Este campo es una construcción teórico-funcional del análisis de los elementos significativos, tanto inmanentes como trascendentes al texto o discurso. Las consideraciones anteriores han tratado de poner de manifiesto la importancia del proceso que va del propio texto hacia el contexto. Esta es la base y, a la vez, la consecuencia del análisis ideológico.

\section{Notas}

1. La corriente contemporánea de análisis ideológico más representativa posiblemente sea la que se ha instaurado alrededor de la figura de Teun A. van Dijk. El lector encontrará importantes sugerencias en su obra Ideología. Un enfoque multidisciplinario. Barcelona: Gedisa. 2000. Para aplicaciones específicas en las ciencias sociales, véanse las obras colectivas siguientes: Teun A. van Dijk. (Comp.) Estudios sobre el discurso. Vol I: El discurso como estructura y proceso. Vol. II: El discurso como interacción social. Barcelona: Gedisa. 2001.

2. La aplicación del análisis ideológico abarca ámbitos tan amplios como los estilos de discurso, la retórica política, la teoría de la argumentación, los géneros y registros, la semiótica discursiva, la propaganda política, la microhistoria. Además, hoy en día también se aplica notablemente en la teoría de la negociación, el análisis de los conflictos internacionales, los estudios sociolingüísticos y de pragmática de la lengua, la cognición social y el diálogo institucional y de las organizaciones, entre otros.

3. Para el concepto de imaginario social y de ideología el lector puede consultar el siguiente trabajo: José Miguel Rodríguez. 1995. "Origen y naturaleza de la teoría política". Revista de Ciencias Jurídicas. ${ }^{\circ}$ 82, Setiembre-diciembre. Sobre el estructuralismo político y la ideología, véase de Vicent Descombes, "Structuralisme". 1996. En: P. Raynaud y S. Rials. Dictionnaire de Philosophie Politique. París: Presses Universitaires de France. 
4. Para ampliar este concepto, véanse los artículos «Structuralisme», y "Structure”; (en): Jean Dubois et al. Dictionnaire de linguistique et de sciences du langage. París, Larouse: 1999.Cf. Eugenio Coseriu. Principios de semántica estructural. Madrid: Gredos, 1977. Cap. 1: Para una semántica diacrónica estructural. Se pueden leer con provecho dos estudios clásicos: de Edmund Leach, Historia del concepto, y de S. Udy, Estructura Social. Ambos en David Sills (Dir.). Enciclopedia internacional de las ciencias sociales. Madrid, Aguilar 1977. Una definición interesante de estructura y estructuralismos en Heinrich Schmidt, Philosophisches Wörterbuch. Stuttgart, Alfred Körner Verlag, 1977.

5. Aunque en la lingüística actual se usa más la expresión "sistema", ambos conceptos, sistema y estructura, se asemejan profundamente. Sin embargo, se dejará la expresión "sistema" sólo para referirse a una estructura formalizada integrada por signos artificiales lógico-matemáticos. En consecuencia, dentro del modelo estructuralista, el sistema es una parte de la estructura. Cf. David Crystal. The Cambridge Encyclopedia of Language. Cambridge, University Press, 1997.

6. "En líneas generales un modelo es un esquema coherente, o sea, una estructura operativa definida, formada por componentes relacionados entre sí, que se emplea para tratar, representar, explicar, definir y justificar un concepto simple, un problema, o un sistema complejo, en suma, cualquier cuestión investigadora o aspecto de la misma. Como la naturaleza del lenguaje es compleja, heteróclita, en palabras de de Saussure, los modelos tienden a simplificarla de una forma operativa y práctica para entenderla mejor, ofreciendo fragmentos de la misma que representan los aspectos más esenciales o los que se juzgan más pertinentes de la investigación que se lleva a cabo de forma precisa y bien delimitada". Alcaráz Varó. Martínez Linares. Op. cit. S. v. Modelo lingüístico.

7. En realidad, existen varios tipos de modelos, los formalizados, es decir, los que son marcos rigurosos, lógico-matemáticos; los modelos funcionales, que destacan las funciones y metafunciones del lenguaje y del discurso; los hipotético-heurísticos, que se utilizan para la investigación; los descriptivos, que tratan de clasificar o establecer taxonomías, etc. Las ciencias sociales suelen ser actualmente ciencias de modelos; tales como la economía, la ciencia política o la geografía humana, para citar sólo algunos ejemplos.

8. El lector interesado en la lingüística saussureana puede consultar con provecho las siguientes obras: E. F. Konrad Koerner. Ferdinand de Saussure. Génesis y evolución de su pensamiento en el marco de la lingüística occidental. Madrid: Gredos. 1982. Georges Mounin. Saussure. Presentación y textos. Barcelona: Anagrama, 1971.

9. Se cita según la traducción de Amado Alonso: Ferdinand de Saussure. Curso de lingüística general. (Madrid, Alianza Editorial, 1983. Publicación póstuma en 1916). Como es sabido, en realidad esta obra está constituida por apuntes de clase recogidos por sus alumnos.

10. También es importante su distinción entre lengua y habla (langue-parole). Desde Saussure es un lugar común afirmar que el habla es el uso individual y concreto que cada hablante hace de la lengua. La lengua es un sistema de signos para la comunicación.

11. Esta teoría está preliminarmente expuesta en su obra, Prolegómenos a una teoría del lenguaje. Madrid: Gredos, 1971. Una exposición sucinta, en: Georges Mounin. La lingüística del siglo XX. Madrid: Gredos. 1984.

12. Recuérdese que el conductismo ejerció un papel preponderante en la psicología y las ciencias de la conducta norteamericana durante la primera mitad del siglo XX y aún después.

13. Bloomfield ha recibido una fuerte influencia de la antropología estructural (Malinowski, Lévi-Strauss). 
14. Lyons sugiere que todos los hombres, cualquiera que sea la cultura en que hayan crecido, están dotados genéticamente de la misma predisposición a la percepción y conceptualización. En virtud de esta predisposición genética, el niño atenderá a unos aspectos más que a otros. Cf. Op. cit. Cap II.

15. En realidad, la versión preliminar de esta obra es del año 1951. Sin embargo, Ullman la rehizo y la publicó en el año 1961 con el nombre de Semantics. An introduction to the science of meaning. Versión castellana: Semántica. Introducción a la ciencia del significado. Madrid: Aguilar Ediciones, S. A. 1967.

16. Coseriu ha influido mucho en la semántica iberoamericana. Para el presente tema deben verse: Eugenio Coseriu. Principios de semántica estructural. Madrid: Gredos, 1977. Eugenio Coseriu. Teoría del lenguaje y lingüística general. Madrid: Gredos, 1982. Para una presentación general de la obra de Coseriu, véase de A. Vilarnovo, Lógica y lenguaje en Eugenio Coseriu. Madrid: Gredos, 1993.

17. En este punto es paradigmática la siguiente obra: A. J. Greimas. 1976. Semántica estructural. Investigación metodológica. Madrid: Gredos. La primera edición es de 1966. Una línea semejante es desarrollada por su discípulo Bernard Pottier. 1977. Lingüística general. Teoría y descripción. Madrid: Gredos.

18. Durante los años sesenta y setenta, el modelo se aplicó profusamente en el análisis de textos literarios, religiosos, jurídicos y políticos.

19. H. Weinreich propuso una "combinatoria semántica", considerada como un intento de integrar la teoría semántica en el cuadro de la sintaxis generativa por parte de la consideración del componente semántico como "condición para la denotación"; es decir, para que un signo pueda ser replicado para designar "algo". También introduce el concepto de "rasgos de transferencia" (transfer feature) mediante el cual es posible explicar los hechos, con base en las restricciones selectivas.

20. Mucho se ha escrito sobre el particular. El lector puede consultar: M. Bierwisch. 1971. El estructuralismo. Historia, problemas, métodos. Barcelona. También: Sobre la clasificación de los rasgos semánticos. (En) Ch. Fillmore y otros. Semántica y sintaxis en la lingüística transformatoria. Madrid: Alianza Editorial. 1976. Un resumen de su teoría en M. Bierwisch. Semántica. (En) John Lyons. (Ed.) Nuevos horizontes de la lingüística. Madrid: Alianza Editorial, 1975.

21. Es conocido que, para Berruto, los indicadores vacíos X e Y señalan, respectivamente, las posiciones en que deben encontrarse el sujeto y el objeto que indican la restricción selectiva. Estas restricciones selectivas coinciden con las "reglas selectivas", que según Chomsky deberían, más bien, formar parte de la sintaxis y no de la semántica; en términos de la gramática generativo-transformacional, deberían formar parte del componente sintáctico y no del componente semántico. Sería, por ejemplo, una regla selectiva la que asigna a "frighten" (asustar) el rasgo (+(+abstracto)- - -(+animado)). Berruto. Idem.

22. Geckeler (1994: 99) usa el término campo léxico (fr. champ lexical, ing. lexical field) para las estructuras lexemáticas. Afirma que: “A este respecto, no tendríamos nada que objetar al uso corriente del término francés champ sémantique, o inglés semantic field, si estos adjetivos expresaran solamente la referencia al estudio de significados exclusivamente léxicos. El término campo lingüístico (fr. champ linguistique, ing. linguistic field) es demasiado amplio para nuestros objetivos, puesto que además del concepto de campo léxico abarca también el de campo sintáctico.” (...) Consideramos el término «campo semántico" (Bedeutungsfeld,) fr. champ sémantique, ing. semantic field- demasiado amplio." A continuación se seguirá la exposición de este autor. 


\section{Bibliografía}

Alcaráz Varó, E. y M. Martínez Linares. 1997. Diccionario de lingüística moderna. Barcelona: Ariel.

Berruto, G. 1974. La semántica. México: Nueva Imagen.

Cristal, David. 1997. The Cambridge Encyclopedia of Language. Cambridge: University Press.

De Saussure, Ferdinand. 1983. Curso de lingüística general. (Trad. Amado Alonso) Madrid: Alianza Editorial.

Descombes, Vincent. 1996. Structuralisme. (En) Raynaud, P. y S. Rials.

Geckeler, Horst. 1994. Semántica estructural y teoría del campo léxico. Madrid: Gredos.

Greimas, A. J. 1976. Semántica estructural. Investigación metodológica. Madrid: Gredos.

Habermas, Jürgen. 1998. Facticidad y validez. Madrid: Trotta.

Hörman, Hans. 1982. Querer decir y entender. Fundamento de una semántica psicológica. Madrid: Gredos.

Katz, Jerrold J. 1979. Teoría semántica. Madrid: Aguilar.

Katz, Jerrold J. y Jerry A. Fodor. 1963. The structure of a semantic theory. Language. 39 (2) (Part 1): 170-210. Existe versión española: La estructura de una teoría semántica. México: Siglo XXI, 1976.

Lyons, John. 1989. Semántica. Barcelona: Teide.

Ogden, C.K. y I. A. Richards. 1984. El significado del significado. Una investigación acerca de la influencia del lenguaje sobre el pensamiento y de la ciencia simbólica. Barcelona: Paidós Ibérica.

Pottier, Bernard. 1977. Lingüística general. Teoría y descripción. Madrid: Gredos.

P. Raynaud et S. Rials. 1996. Dictionnaire de Philosophie Politique. París: Presses Universitaires de France.

Sapir, E. 1984. El lenguaje. Introducción al estudio del habla. México: Fondo de Cultura Económica.

Schmidt, Heinrich. 1977. Philosophisches Wörterbuch. Stuttgar: Alfred Körner Verlag. 
Ullman, S.1967. Semántica. Introducción a la ciencia del significado. Madrid: Aguilar Ediciones.

Van Dijk, T. A. 2000. Ideología. Un enfoque multidisciplinario. Barcelona: Gedisa. 